TRANSACTIONS OF THE

AMERICAN MATHEMATICAL SOCIETY

Volume 348, Number 5, May 1996

\title{
A CLASS OF COUNTEREXAMPLES TO THE GEL'FAND-KIRILLOV CONJECTURE
}

\author{
JACQUES ALEV, ALFONS OOMS, AND MICHEL VAN DEN BERGH
}

\begin{abstract}
Let $G$ be a connected non-special semisimple algebraic group and let $W$ be a finite dimensional $G$-representation such that $W$ has trivial generic stabilizer. Let $\mathfrak{g}=\operatorname{Lie}(G)$. Then the semi-direct product $\mathfrak{g} \oplus W$ is a counterexample to the $\mathrm{Gel}^{\prime}$ fand-Kirillov conjecture.
\end{abstract}

\section{IntroduCtion AND STATEMENT OF THE MAIN RESUlT}

In the sequel $k$ will be an algebraically closed field of characteristic zero. All objects we consider are $k$-objects. For a Lie algebra $L$ over $k$ we denote by $U(L)$ its universal enveloping algebra and by $D(L)$ the field of fractions of $U(L)$. In [3] Gel'fand and Kirillov formulate the following conjecture :

Conjecture (GK). Assume that $L$ is a finite dimensional algebraic Lie algebra over $k$. Then $D(L)$ is a Weyl skew field over a purely transcendental extension of $k$.

This conjecture is known to be true for $L$ solvable [2], [7], [12] and for semisimple Lie algebras of type $A_{n}[3]$. It is open for the other types.

In this note we construct a class of counterexamples to (GK). To the best of our knowledge, these are the first such examples. However in [11] counterexamples to (GK) are given over a non-algebraically closed base field. These are given by the non-trivial twisted versions of $\mathfrak{s l}_{2}$.

Before we outline our construction let us mention that in [4] Gel'fand and Kirillov prove that for semisimple $L, D(L)$ becomes a Weyl skew field after extending the center. This suggests the following weakening of (GK).

Conjecture $\left(\mathbf{G K}^{\prime}\right)$. Assume that $L$ is a finite dimensional algebraic Lie algebra over $k$. Then there exists a field extension $K$ of the center of $D(L)$, purely transcendental over $k$ such that the field of fractions of $D(L) \otimes_{Z(D(L))} K$ is a Weyl skew field.

The examples we give are counterexamples to (GK) but not to $\left(\mathrm{GK}^{\prime}\right)$ (see Corollary 2.5). In fact the authors are rather convinced that $\left(\mathrm{GK}^{\prime}\right)$ is true.

Now we describe our examples. Let $G$ be a connected semisimple algebraic group over $k$ and let $W$ be a finite dimensional $G$-representation. Let $\mathfrak{g}=\operatorname{Lie}(G)$. Then

Received by the editors February 22, 1995.

1991 Mathematics Subject Classification. Primary 17B35; Secondary 16K40.

Key words and phrases. Gel'fand-Kirillov conjecture.

The third author is a senior researcher at the NFWO. 
$W$ is also a $\mathfrak{g}$-representation. Let $L$ be the semidirect product $\mathfrak{g} \oplus W$. That is, if $(u, w),\left(u^{\prime}, w^{\prime}\right) \in \mathfrak{g} \oplus W$ then

$$
\left[(u, w),\left(u^{\prime}, w^{\prime}\right)\right]=\left(\left[u, u^{\prime}\right], u w^{\prime}-u^{\prime} w\right) .
$$

Examples of this type had been considered earlier in connection with the Gel'fand Kirillov conjecture by Nghiem-Xuan Hai [5] and by the first two authors.

Now recall that $G$ is called special if any principal homogeneous $G$-space is locally trivial for the Zariski topology. The following is our main result :

Theorem 1.1. Assume that the generic stabilizer of $W$ is trivial and let $L=\mathfrak{g} \oplus W$ be as above. Then the following are equivalent:

(1) $D(L)$ is a Weyl skew field over some field.

(2) $G$ is special.

The proof follows by combining Corollaries 2.3 and 4.3 below.

The smallest counterexample to (GK) that can be made with Theorem 1.1 is given by $\mathfrak{g}=\mathfrak{s l}_{2}$ and $W$ equal to two copies of the adjoint representation of $\mathfrak{g}$. Then $\operatorname{dim} L=9$. This should be compared with work of the first two authors which shows (by explicit computation) that (GK) holds for all Lie algebras of dimension $\leq 6$.

The authors wish to thank Thierry Levasseur and Jean-Louis Colliot-Thélène for some useful discussions. We also thank Hanspeter Kraft for allowing us to include some explicit results of his in the case $G=\mathrm{Sl}_{2}$ (see Appendix).

\section{An alternative Description of $D(L)$}

We let $G, W, L$ etc $\ldots$ be as above. Let $K, F$ be respectively the function fields of $W^{*}$ and $W^{*} / / G$. Of course $F=K^{G}$. Let $X$ be the generic fiber of $W^{*} \rightarrow W^{*} / / G$. If the generic stabilizer of $W$ is trivial then it follows from [13, Appendix], the Luna Slice Theorem and Lemma 2.6 below that $X$ is a principal homogeneous $G$-space.

Our first step is the following observation :

Proposition 2.1. Assume that the generic stabilizer of $W$ is finite. Then $D(L)$ is isomorphic to the field of fractions of $\mathcal{D}_{F}(K)$, the ring of differential operators on $K / F$.

Proof. Let $l$ be a field. If $\mathfrak{h}$ is an $l$-Lie algebra acting on an $l$-algebra $A$ by derivations then by definition $A \#_{l} U(\mathfrak{h})$ is the algebra which equals $A \otimes_{l} U(\mathfrak{h})$ as $l$-vector space and whose multiplication is defined by

$$
(a \# u)\left(a^{\prime} \# u^{\prime}\right)=a\left(u a^{\prime}\right) \# u u^{\prime}
$$

where $a, a^{\prime} \in A, u, u^{\prime} \in \mathfrak{h}$.

If $A$ is commutative then there is a canonical $l$-algebra homomorphism

$$
A \#_{l} U(\mathfrak{h}) \rightarrow \mathcal{D}_{l}(A)
$$

and we have the following easy lemma :

Sublemma. Suppose that $A$ is commutative and smooth over $l$. Assume furthermore that the canonical map $A \otimes_{l} \mathfrak{h} \rightarrow \operatorname{Der}_{l}(A)$ is an isomorphism. Then the map given by (2.1) is an isomorphism. 
Proof. The PBW-filtration on $U(\mathfrak{h})$ extends to a filtration on $A_{\#_{l}} U(\mathfrak{h})$ such that $\operatorname{gr} A \#_{l} U(\mathfrak{h})$ is equal to the symmetric algebra $S_{A}\left(A \otimes_{l} \mathfrak{h}\right)$.

Similarly $\mathcal{D}_{l}(A)$ is filtered by order of differential operators and since $A / l$ is assumed to be smooth, gr $\mathcal{D}_{l}(A)=S_{A}\left(\operatorname{Der}_{l}(A)\right)$. This finishes the proof since (2.1) is compatible with these two filtrations.

It is easily verified that one has the identity

$$
U(L)=S W \#_{k} U(\mathfrak{g}) .
$$

So $D(L)$ is the field of fractions of $K \#_{k} U(\mathfrak{g})=K \#_{F} U\left(\mathfrak{g}_{F}\right)$ where $\mathfrak{g}_{F}=F \otimes_{k} \mathfrak{g}$. The proposition now follows from the above sublemma and the next one.

Sublemma. The canonical map

$$
K \otimes_{F} \mathfrak{g}_{F} \rightarrow \operatorname{Der}_{F}(K)
$$

is an isomorphism.

Proof. First note that the generic stabilizer of $W^{*}$ is also finite (Lemma 2.6 below) and hence $\operatorname{tr} . \operatorname{deg} K / F=\operatorname{dim} G=\operatorname{dim} \mathfrak{g}_{F}$. Thus the left- and right-hand sides of (2.2) are $K$-vector spaces of the same dimension. So it suffices to prove that (2.2) is injective. To this end it suffices to prove that

$$
K \otimes_{k} \mathfrak{g} \rightarrow \operatorname{Der}_{k}(K)
$$

is injective. Let $u_{1}, \ldots, u_{n}$ be a basis for $\mathfrak{g}$ and let $u=\sum_{i} f_{i} u_{i}$ be a non-zero element in the kernel of (2.3). Choose $x \in W^{*}$ in such a way that

- the stabilizer of $x$ is finite;

- all $f_{i}$ are regular in $x$;

- not all $f_{i}$ are zero in $x$.

Then $u(x)$ defines a non-zero element of the kernel of the differential in $e$ of the map $G \rightarrow W^{*}: g \mapsto g x$. Now the stabilizer of $x$ is finite and so this map is an immersion locally around $e$. Therefore the differential is injective in $e$ which yields a contradiction.

Corollary 2.2. Assume that the generic stabilizer of $W$ is finite. Then the center of $D(L)$ is equal to $F$.

Proof. One easily verifies from Proposition 2.1 that the center of $D(L)$ is given by those elements of $K$ that are constant for all $F$-derivations of $K$. Because $\mathfrak{g}$ maps to $\operatorname{Der}_{F}(K)$ it is sufficient to show that $K^{\mathfrak{g}}=F$. Now the fact that $G$ is connected implies that $K^{\mathfrak{g}}=K^{G}=F$.

We are now in a position to prove half of Theorem 1.1.

Corollary 2.3. $D(L)$ is a Weyl skew field over $F$ in the following cases.

(1) The generic stabilizer of $W$ is finite and $K / F$ is purely transcendental.

(2) The generic stabilizer of $W$ is trivial and $G$ is special.

Proof. (1) is a direct application of Proposition 2.1. In case (2), $X$ is a principal homogeneous $G$-space and hence the fact that $G$ is special implies that $X \cong G_{F}$. This shows what we want since $G$ is rational. 
Example 2.4. Let $\mathfrak{g}=\mathfrak{s l}_{2}$ and let $W$ be either the 4- or 5-dimensional irreducible representation of $\mathfrak{g}$. Then the generic stabilizer of $W$ is non-trivial so Corollary 2.3(2) does not apply. However Kraft shows by explicit computation that in this case $K / F$ is rational (see Appendix). So 2.3(1) yields that $D(L)$ is a Weyl skew field.

Corollary 2.5. Assume that the generic stabilizer of $W$ is trivial. Then the field of fractions of $D(L) \otimes_{F} K$ is a Weyl skew field over $K$.

Proof. In this situation $X$ is a principal homogeneous $G$-space. Now the generic point of $X$ is by definition a $K$-rational point and so we have $X_{K} \cong G_{K}$. In particular the field of fractions of $K \otimes_{F} K$ is purely transcendental over $K$. The corollary now follows from Proposition 2.1, and the fact that differential operators are compatible with base field extension.

In Theorem 1.1 and Proposition 2.1 we have stated, for elegance, the results in terms of $W$, although it would have been more natural to work with $W^{*}$. We therefore recall the following well-known result.

Lemma 2.6. Let $G$ be a connected semisimple algebraic group over $k$. Then there exists a (non-unique) automorphism $\theta$ of $G$ such that for any finite dimensional representation $W$ of $G$ one has $W^{*}={ }_{\theta} W$.

Proof. There are various constructions for $\theta$. One possibility is the following. Let $T \subset G$ be a maximal torus and let $\theta^{\prime}$ be the automorphism of $T$ which sends $t$ to $t^{-1}$. $\theta^{\prime}$ sends the roots of $(G, T)$ to their inverses and hence defines an automorphism of the root system of $(G, T)$. Therefore $\theta^{\prime}$ extends to an automorphism $\theta$ of $G[6$, Chapter 32]. This $\theta$ is the one we need since it sends the character of $W$ to that of $W^{*}$.

\section{Subfields of WeYl SKeW FIELDS}

We now need a criterion for certain division algebras not to be Weyl skew fields. We use the following observation :

Proposition 3.1. Assume that $D$ is a Weyl skew field with center $F$. Let $K \subset D$ be a subfield containing $F$ and let $H$ be a reductive group over $F$. Then the canonical map

has trival kernel.

$$
H_{\mathrm{et}}^{1}(F, H) \rightarrow H_{\mathrm{et}}^{1}(K, H)
$$

(Recall that for any field $F, H_{\mathrm{et}}^{1}(F, H)$ is a pointed set parametrizing the principal homogeneous $H$-spaces over $F$.)

Proof. By definition $D$ is the field of fractions of some Weyl algebra $A_{n}$. If we filter $A_{n}$ with the Bernstein filtration then gr $A_{n}$ is a graded polynomial ring in $2 n$ variables, each of degree one.

The filtration on $A_{n}$ extends to a filtration on $D$ whose associated graded ring is the graded field of fractions [14] of gr $A_{n}$. It is easy to see that this implies that gr $D=P\left[X, X^{-1}\right]$ where $P$ is a purely transcendental field extension of $F$ and $\operatorname{deg} X=1$.

One easily verifies that the filtration on $D$ is obtained from a discrete valuation with residue field $P$ [10]. Then the restriction of this discrete valuation to $K$ is 
either trivial or also a discrete valuation. In the first case $K \subset P$ and in the second case the residue field of the valuation on $K$ is contained in $P$.

It now suffices to invoke the following lemma.

Sublemma. Assume that $K / F$ is a field extension which satisfies one of the following conditions.

(1) $K / F$ is unirational.

(2) $K$ is the field of fractions of a discrete valuation ring $R$ containing $F$ whose residue class field is unirational.

Then $H_{\mathrm{et}}^{1}(F, H) \rightarrow H_{\mathrm{et}}^{1}(K, H)$ has trivial kernel.

Proof. We only prove (2) since there is an extra point involved.

Let $l$ be the residue class field of $R$ and let $P / F$ be a purely transcendental extension containing $l$.

Let $X \in \operatorname{ker}\left(H_{\mathrm{et}}^{1}(F, H) \rightarrow H_{\mathrm{et}}^{1}(K, H)\right)$. By [15], $H_{\mathrm{et}}^{1}(R, H) \rightarrow H_{\mathrm{et}}^{1}(K, H)$ has trivial kernel, so

$$
X \in \operatorname{ker}\left(H_{\mathrm{et}}^{1}(F, H) \rightarrow H_{\mathrm{et}}^{1}(R, H)\right) .
$$

But then $X$ is also in the kernel of the composition

$$
H_{\mathrm{et}}^{1}(F, H) \rightarrow H_{\mathrm{et}}^{1}(R, H) \rightarrow H_{\mathrm{et}}^{1}(l, H) \rightarrow H_{\mathrm{et}}^{1}(P, H) .
$$

Now the map $H_{\text {et }}^{1}(F, H) \rightarrow H_{\text {et }}^{1}(P, H)$ is injective since $P / F$ is purely transcendental and hence $X$ must be trivial.

This finishes the proof of Proposition 3.1.

\section{NON-SPECIAL GROUPS}

To conclude we need the following result due to Luna (unpublished).

Lemma 4.1. Let $G$ be a non-special semisimple algebraic group and let $V$ be a $G$-representation with trivial generic stabilizer. Then $V \rightarrow V / / G$ is generically a principal homogeneous $G$-space which is not trivial.

Proof. This follows directly from [9, IV.2.4]. However we will take the opportunity here to present a slightly different proof which perhaps has some independent interest.

Let $G$ be a semisimple, not necessarily non-special, algebraic group. According to [13, Appendix], the generic orbit in $V$ is closed, so by the Luna Slice Theorem there exists a non-empty open affine $G$-invariant subset $U$ of $V$ such that the action of $G$ on $U$ is free.

Now recall that a principal homogeneous $G$-space $\phi: Y \rightarrow X$ is called versal if for all local rings $A$ and for all principal homogeneous $G$-spaces $\theta: Z \rightarrow \operatorname{Spec} A$ there is a morphism Spec $A \rightarrow X$ such that the pullback of $\phi$ along this map is equal to $\theta$.

To prove the proposition it now suffices to prove the following stronger result which was suggested to us by Colliot-Thélène.

Theorem 4.2. Let $G$ be a linear algebraic group over $k$ and let $V$ be a non-empty finite dimensional $G$-representation. Assume that there is a non-empty open $U \subset V$ such that there exists a geometric quotient $U \rightarrow U / G$ which is a principal homogeneous $G$-space. Then $U \rightarrow U / G$ is versal. 
Proof. It is shown by Serre [17] that if $G \subset \mathrm{Gl}_{n}$ is an embedding then $\mathrm{Gl}_{n} \rightarrow \mathrm{Gl}_{n} / G$ is versal. We now consider the following diagram

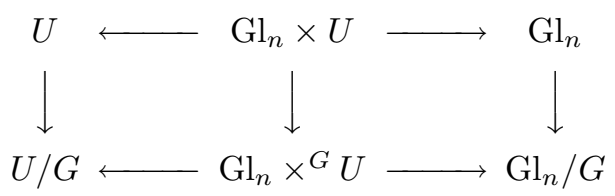

Here the left and right squares are pull backs. Furthermore $\mathrm{Gl}_{n} \times{ }^{G} U$ is open in $\mathrm{Gl}_{n} \times{ }^{G} V$ which is a vector bundle on $\mathrm{Gl}_{n} / G$.

It is easy to deduce from this last fact that the map $\mathrm{Gl}_{n} \times{ }^{G} U \rightarrow \mathrm{Gl}_{n} / G$ has sections locally and is therefore surjective on $A$-points (where, as above, $A$ denotes a local ring over $k$ ). Hence an $A$-point of $\mathrm{Gl}_{n} / G$ may be lifted to $\mathrm{Gl}_{n} \times{ }^{G} U$ and then projected to $U / G$. This shows that any pullback of $\mathrm{Gl}_{n}$ for a map Spec $A \rightarrow \mathrm{Gl}_{n} / G$ can be realized as a pullback of $U$ for a map $\operatorname{Spec} A \rightarrow U / G$. This is what we had to show.

Corollary 4.3. Let the notation be as in the statement of Theorem 1.1. If $G$ is not special then $D(L)$ is not a Weyl skew field.

Proof. By 4.1, $X$ is a non-trivial principal homogeneous $G$-space. Now $X$ is trivially split by $K$ and hence $X$ represents a non-trivial element of

$$
\operatorname{ker}\left(H_{\mathrm{et}}^{1}(F, G) \rightarrow H_{\mathrm{et}}^{1}(K, G)\right) .
$$

By Corollary 2.2 the center of $D(L)$ is $F$. Then, by Proposition 3.1, $D(L)$ cannot be a Weyl skew field over $F$ since it contains $K$.

\section{Appendix A. Some explicit computations in the CaSe $\mathrm{Sl}_{2}$.}

The results in this appendix are due to Kraft and we are grateful for his permission to include them here. We will sketch a proof of the following result.

Proposition A.1. Let $G=\mathrm{Sl}_{2}$ and let $W$ be either the 4- or 5-dimensional irreducible representation of $G$. Then $k(W) / k(W)^{G}$ is purely transcendental.

Proof. Assume first that $\operatorname{dim} W=5$. It is classical that $k[W]^{G}$ is a polynomial ring with two generators, say $A, H$, of degree two and three respectively $[16$, p. 80]. Let $\Gamma$ be the generic stabilizer of $W$. It is a binary dihedral group of order eight, generated by a diagonal matrix of order 4 and $\left(\begin{array}{cc}0 & 1 \\ -1 & 0\end{array}\right)$.

Let $\pi: W \rightarrow W / / G$ be the quotient map and let $U \subset W / / G$ be the generic Luna stratum. Then $\pi^{-1}(U) \rightarrow U$ is a fiber bundle with fiber $G / \Gamma$.

The multiplicative group $k^{*}$ acts by scalar multiplication on $W$ and $W / / G$. Since this action commutes with $G, k^{*}$ preserves $U$ and $\pi^{-1}(U)$. Put $W^{\prime}=\pi^{-1}(U) \backslash$ $\{\{A=0\} \cup\{H=0\}\}, Q=\pi\left(W^{\prime}\right)$. By construction the $k^{*}$-action is free on $W^{\prime}$ and $Q$ and we get a pull-back diagram

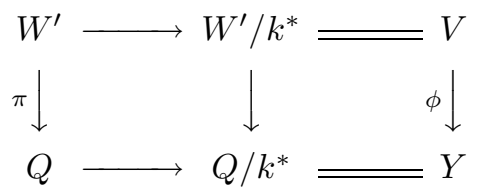

Thus it suffices to prove that $\phi: V \rightarrow Y$ is purely transcendental. By construction $\phi: V \rightarrow Y$ is the quotient by $G$ and is a fiber bundle with fiber $G / \Gamma$. Moreover $Y$ 
is an affine smooth rational curve. We can factor $\phi$ in the following form

$$
V \stackrel{\phi_{1}}{\rightarrow} V / U \stackrel{\phi_{2}}{\rightarrow} V / B \stackrel{\phi_{3}}{\rightarrow} Y
$$

where $B, U$ denote as usual the subgroups of upper triangular and unipotent upper triangular matrices in $G$. The first two maps are quotients by, respectively, the one-dimensional additive and multiplicative groups, hence these are locally trivial in the Zariski-topology. The last map $\phi_{3}$ is a fiber bundle with fiber $B \backslash G / \Gamma \cong \mathbb{P}^{1}$ and it is well-known that every $\mathbb{P}^{1}$-bundle over a smooth curve is locally trivial in the Zariski-topology (Theorem of Noether-Enriques, cf. [1, Theorem III.4]). Hence we are done.

The case $\operatorname{dim} W=4$ is similar but easier. Since we now have $\operatorname{dim} k[W]^{G}=1$ we can invoke Noether-Enriques directly. That is we do not have to divide out the $k^{*}$-action.

Remark A.2. The quotient $W^{\prime} \rightarrow Q$ in the above proof is not locally trivial in the Zariski-topology since otherwise there would be some affine open subset of $W^{\prime}$, isomorphic to $G / \Gamma \times$ something. Since $G / \Gamma$ is not factorial, this is impossible. $\left(\operatorname{Pic}(G / \Gamma) \cong(\mathbb{Z} / 2 \mathbb{Z})^{2}\right.$, see $[8, \S 3$, Proposition 3.2].)

\section{REFERENCES}

1. A. Beauville, Surface algébriques complexes, Astérisque, vol. 54, Soc. Math. France, 1978. MR 58:5686

2. W. Borho, P. Gabriel, and R. Rentschler, Primideale in Einhüllenden auflösbarer LieAlgebren, Lecture Notes in Math., vol. 357, Springer, Berlin and New York, 1973. MR 51:12965

3. I. M. Gel'fand and A. A. Kirillov, Sur les corps liés aux algèbres enveloppantes des algèbres de Lie, Inst. Hautes Études Sci. Publ. Math. 31 (1966), 5-19. MR 34:7731

4. _ The structure of the Lie field connected with a split semisimple Lie algebra, Funktsional. Anal. i Prilozhen. 3 (1969), 6-21. MR 39:2827

5. N.-X. Hai, Réduction de produits semi-directs et conjecture de Gel' fand et Kirillov, Bull. Soc. Math. France 107 (1979), 241-267. MR 80i:17013

6. J. E. Humpreys, Linear algebraic groups, Graduate Texts in Math., vol. 21, Springer Verlag, Berlin and New York, 1975. MR 53:633

7. A. Joseph, Proof of the Gel'fand-Kirillov conjecture for solvable Lie algebras, Proc. Amer. Math. Soc. 45 (1974), 1-10. MR 52:522

8. F. Knop, A. Kraft, and T. Vust, The Picard group of a G-variety, Algebraische Transformationsgruppen und Invariantentheorie, DMV-Seminar, vol. 13, Birkhäuser Verlag, 1989, pp. 7787. CMP 90:09

9. H. Kraft and G. Schwartz, Reductive group actions with one-dimensional quotient, Inst. Hautes Études Sci. Publ. Math. 76 (1992), 1-97. MR 94e:14065

10. H. Li and F. Van Oystaeyen, Filtrations on simple artinian rings, J. Algebra 132 (1990), 361-376. MR 91g:16034

11. P. Malcolmson, Enveloping algebras of simple three-dimensional Lie algebras, J. Algebra 146 (1992), 210-218. MR 93c:17017

12. J. C. McConnell, Representations of solvable Lie algebras and the Gel'fand-Kirillov conjecture, Proc. London Math. Soc. (3) 29 (1974), 453-484. MR 50:9997

13. D. Mumford and J. Fogarty, Geometric invariant theory, Ergebnisse der Mathematik und ihrer Grenzgebiete, vol. 34, Springer Verlag, Berlin, Heidelberg, New York, 1982. MR 86a:14006

14. C. Nastacescu and F. Van Oystaeyen, Graded ring theory, North-Holland, 1982. MR 84i:16002

15. E. A. Nisnevich, Espaces homogènes principaux rationellement triviaux et arithmétique des schémas en groupes réductifs sur les anneaux de Dedekind, C. R. Acad. Sci. Paris Sér. I Math. 299 (1984), 5-8. MR 86f:14034 
16. I. Schur, Vorlesungen über Invariantentheorie, Grundlehren Math. Wiss., vol. 143, Springer Verlag, 1968. MR 37:5248

17. J.-P. Serre, Espaces fibrés algébriques, Anneaux de Chow et Applications, Séminaire Chevalley, vol. 2, Secrétariat Mathématiques, 1958.

(J. Alev) Université de Reims, UfR des Sciences, Département de Mathématiques, Moulin de la Housse, BP 347, 51062 Reims Cedex

E-mail address: jle@ccr.jussieu.fr, jacques.alev@cleo.univ-reims.fr

(A. Ooms and M. Van den Bergh) Limburgs Universitair Centrum, Departement Wni, Universitaire Campus, 3590 Diepenbeek, Belgium

E-mail address, A. Ooms: aooms@luc.ac.be

E-mail address, M. Van den Bergh: vdbergh@luc.ac.be 